\title{
DETERMINAÇÃO DE HORMÔNIOS ESTRÓGENOS EM ÁGUA POTÁVEL USANDO CLAE-DAD
}

\author{
Raphael Teixeira Verbinnen* e Gilvanda Silva Nunes \\ Departamento de Tecnologia Química, Centro de Ciências Exatas e Tecnologia, Universidade Federal do Maranhão, Av. dos \\ Portugueses, s/n, 65080-040 São Luís - MA, Brasil
}

Eny Maria Vieira

Instituto de Química de São Carlos, Universidade de São Paulo, Av. Trabalhador Sãocarlense, 400, 13566-590

São Carlos - SP, Brasil

Recebido em 14/9/09; aceito em 2/6/10; publicado na web em 24/8/10

\begin{abstract}
DETERMINATION OF ESTROGENS IN DRINKING WATER USING HPLC-DAD. An analytical procedure for determination of estriol, 17ß-estradiol, estrone and 17 $\alpha$-ethinylestradiol in drinking water is presented. The method employs solid phase extraction (SPE) and sample dechlorination as cleanup procedures, followed by HPLC-DAD analysis. Validation was carried out using RE No. 899/2003 guidelines established by the Agência Nacional de Vigilância Sanitária (National Agency of Sanitary Surveillance, Brazil), with some adaptations. The statistically evaluated results have shown that the method is selective, precise $(0,06 \%$ to $19,40 \% \mathrm{CV})$ and accurate ( $91,52 \%$ to $109,41 \%$ average recoveries). The developed method was applied to the analysis of these contaminants in drinking water from São Luís, MA.
\end{abstract}

Keywords: estrogen hormones; HPLC-DAD; water supply.

\section{INTRODUÇÃO}

Desreguladores endócrinos (DE) são definidos como substâncias exógenas capazes de causar efeitos adversos à saúde, interferindo no sistema endócrino de organismos aquáticos, e nos últimos anos têm sido um dos principais tópicos de pesquisa em diferentes áreas do conhecimento. ${ }^{1}$

O estrógeno sintético $17 \alpha$-etinilestradiol (EE2), amplamente usado na medicina em terapias de reposição e métodos contraceptivos, e os naturais estrona (E1), 17ß-estradiol (E2) e estriol (E3) são considerados como responsáveis pela maioria dos efeitos de desregulação endócrina, ${ }^{2-4}$ pois afeta o sistema endócrino de peixes em concentrações de $1 \mathrm{ng} \mathrm{L}^{-1}, 5$ podendo ser encontrados no ambiente em concentrações na ordem de $\mu \mathrm{g} \mathrm{L}^{-1}$ e ng $\mathrm{L}^{-1}{ }^{16,7}$

A persistência da atividade dos estrogênios tem origem na contínua introdução em ambientes aquáticos por meio da disposição inadequada de esgoto sanitário e industrial, como também pelo uso de lodo ativado de estação de tratamento de esgoto na agricultura. ${ }^{2,8}$

O uso de águas superficiais para o consumo humano, bem como a transformação de seus mananciais como receptores de esgotos sanitários, tratados ou não, têm sido os principais motivos de preocupação acerca da contaminação das fontes de água. ${ }^{9}$

Diante da problemática de poluição dos compartimentos aquáticos que servem de fonte de captação de água para o abastecimento público, há consideráveis possibilidades de contaminação também da água potável, por esses estrógenos, já que no Brasil o índice de tratamento de esgotos ainda é baixo, principalmente na Região Nordeste. ${ }^{10}$ Além disso, os tratamentos convencionais de água e de esgoto sanitário não são capazes de remover completamente tais substâncias, permanecendo ainda no meio aquático. ${ }^{7,11,12}$

Diversos trabalhos têm sido dedicados a desenvolver métodos para determinação e quantificação de desreguladores endócrinos e avaliação de sua atividade estrogênica em organismos aquáticos. ${ }^{1}$ Técnicas de preparo de amostras (SPE, SPME) e cromatográficas (LC, GC) com diferentes detectores (DAD, FLD, MS) $)^{8,13,14}$ e outras

*e-mail: raphaeltv@hotmail.com fundamentadas em testes toxicológicos (YES, ELISA, E-screen, ELRA) ${ }^{14,15}$ têm sido desenvolvidas.

Estudos conduzidos por pesquisadores de Campinas, SP, apontaram a presença de hormônios estrógenos no Rio Atibaia, que abastece $92 \%$ do município. ${ }^{16}$ Em 2006, Sodré e colaboradores ${ }^{17}$ identificaram, neste mesmo ecossistema aquático, em um ponto a jusante da cidade de Campinas, níveis de $17 \beta$-estradiol e $17 \alpha$-etinilestradiol que chegaram a 2,51 e $0,31 \mu \mathrm{g} \mathrm{L} \mathrm{L}^{-1}$, respectivamente.

Em São Luís, MA, a água potável que é distribuída à população é proveniente de diferentes mananciais. O rio Itapecuru, que recebe efluentes de cerca de 3 milhões de pessoas e cuja estação de tratamento de água (ETA) dista $56 \mathrm{Km}$ da rede de distribuição, ${ }^{18} \mathrm{e}$ o Reservatório do Batatã, que recebe impactos antrópicos de mais de 40 mil pessoas, ${ }^{19}$ são as principais fontes de água superficial. Até o momento, nenhum estudo objetivando verificar se está ocorrendo contaminação por hormônios estrógenos foi realizado nessas áreas, não obstante a premente necessidade. Isso se deve, de uma parte, à carência de laboratórios que se proponham a otimizar métodos analíticos e torná-los procedimentos de rotina, e de outra, à falta de pessoal com formação em análise desses poluentes.

Este trabalho objetivou desenvolver um método analítico que fosse, a um só tempo, sensível, simples e relativamente rápido, voltado para análise de hormônios estrógenos mais comumente encontrados em amostras de água. Posteriormente, pretende-se empregá-lo em monitoramentos rotineiros e em estudos ecotoxicológicos na região.

\section{PARTE EXPERIMENTAL}

\section{Reagentes e soluções}

Todos os padrões de estrógenos, com pureza mínima de 97\%, foram obtidos da Sigma-Aldrich e da Fluka. Os solventes orgânicos de grau cromatográfico, metanol $(\mathrm{MeOH})$, acetonitrila $(\mathrm{ACN})$ e acetona (ACT), foram obtidos da J.T.Baker. Água desionizada foi obtida a partir dos sistemas Milli- e Direct-Q, da Millipore e o gás nitrogênio, com 99,999\% de pureza, da White Martins. Da Agilent, foram obtidos cartuchos de extração em fase sólida do tipo seringa, 
Tabela 1. Procedimentos de extração usados neste estudo

\begin{tabular}{|c|c|c|c|c|}
\hline \multirow[t]{2}{*}{ Etapa } & \multicolumn{4}{|c|}{ Extração em Fase Sólida (EFS) } \\
\hline & $\mathrm{P}-1$ & $\mathrm{P}-2$ & $\mathrm{P}-3$ & $\mathrm{P}-4$ \\
\hline Condicionamento & $\begin{array}{c}5 \mathrm{~mL} \mathrm{MeOH}^{\mathrm{a}} ; 7 \mathrm{~mL} \mathrm{ACN}^{\mathrm{b}} ; \\
5 \mathrm{~mL} \mathrm{H}_{2} \mathrm{O}\end{array}$ & $\begin{array}{l}9 \mathrm{~mL} \mathrm{MeOH} \\
9 \mathrm{~mL} \mathrm{H}_{2} \mathrm{O}\end{array}$ & $\begin{array}{c}6 \mathrm{~mL} \mathrm{Hex}^{\mathrm{c}} ; 2 \mathrm{~mL} \mathrm{ACT}{ }^{\mathrm{d}} \\
6 \mathrm{~mL} \mathrm{MeOH} ; 10 \mathrm{~mL} \mathrm{H}_{2} \mathrm{O} \mathrm{pH} 3\end{array}$ & $\begin{array}{c}2 \mathrm{~mL} \mathrm{ACT} ; 6 \mathrm{~mL} \mathrm{MeOH} ; \\
10 \mathrm{~mL} \mathrm{H}_{2} \mathrm{O} \mathrm{pH} 3\end{array}$ \\
\hline Adição de amostra & $200 \mathrm{~mL}$ & $200 \mathrm{~mL}$ & $200 \mathrm{~mL}$ & $200 \mathrm{~mL}$ \\
\hline Lavagem & $5 \mathrm{~mL}$ água & $10 \mathrm{~mL} \mathrm{MeOH}: \mathrm{H}_{2} \mathrm{O}(1: 9)$ & 10 mL MeOH: $\mathrm{H}_{2} \mathrm{O}(1: 9)$ & 10 mL MeOH: $\mathrm{H}_{2} \mathrm{O}(1: 9)$ \\
\hline Eluição & $6 \mathrm{~mL} \mathrm{ACN}$ & $2 \times 2 \mathrm{~mL} \mathrm{ACT}$ & $4 \mathrm{~mL} \mathrm{ACT}$ & $4 \mathrm{~mL} \mathrm{ACT}$ \\
\hline Secagem do extrato & nitrogênio & nitrogênio & nitrogênio & nitrogênio \\
\hline Reconstituição & $0,5 \mathrm{~mL} \mathrm{MeOH}$ & $0,5 \mathrm{~mL} \mathrm{MeOH}$ & $0,5 \mathrm{~mL} \mathrm{ACN}$ & $0,5 \mathrm{~mL}$ ACN \\
\hline
\end{tabular}

${ }^{\mathrm{a}}$ Metanol; ${ }^{\mathrm{b}}$ Acetonitrila; ${ }^{\mathrm{c}}$ Hexano; ${ }^{\mathrm{d}}$ Acetona.

séries AccuBondII ODS-C18 (500mg, 6 mL) e SampliQ OPT (150 $\mathrm{mg}, 6 \mathrm{~mL}$ ). Para acidificação da fase móvel, foi empregado o ácido acético glacial P.A., com pureza 99,9\% (Merck).

Solução aquosa de metabissulfito de sódio (Sigma-Aldrich) foi preparada a uma concentração de $3 \%(\mathrm{~m} / \mathrm{v})$. As soluções-padrão estoque dos hormônios estrógenos [estriol (E3), $17 \beta$-estradiol (E2), estrona (E1) e $17 \alpha$-etinilestradiol (EE2)] foram preparadas em $\mathrm{MeOH}$ em uma concentração de $100 \mathrm{mg} \mathrm{L}^{-1}$. A partir destas, foram obtidas as soluções de trabalho, mediante diluição com MeOH. Todas as soluções preparadas foram acondicionadas em frasco âmbar e armazenadas em refrigerador a $4{ }^{\circ} \mathrm{C}$.

Todas as vidrarias foram lavadas com água e detergente comum, deixadas de molho em uma solução a 5\% (v/v) de Extran alcalino (Merck) por cerca de $12 \mathrm{~h}$, e em seguida enxaguadas seqüencialmente com água de torneira e água destilada, antes do uso.

\section{Extração dos analitos}

Foram testados quatro procedimentos de EFS, um (P-1) segundo descrito por Araújo ${ }^{3}$ e outro (P-3) de acordo com Lopes..$^{20}$ Ambos os trabalhos empregaram cartuchos preenchidos com $\mathrm{C}_{18}$ para a extração. Os demais procedimentos, denominados P-2 e P-4 (Tabela 1), foram propostos a partir destes, buscando-se empregar solventes de baixos custo e toxicidade.

Alda e Barceló ${ }^{6}$ indicaram bons resultados de recuperação ao aplicar o adsorvente polimérico (Oasis HLB, Waters) para a extração dos quatro estrógenos aqui investigados, portanto incluiu-se no estudo a extração empregando um adsorvente polimérico similar (OPT, da Agilent), usando-se os mesmos procedimentos descritos na Tabela 1, para comparação das performances de cada material adsorvente.

Os fluxos foram de $3 \mathrm{~mL} \mathrm{~min}^{-1}$ no condicionamento, de $2 \mathrm{~mL}$ $\min ^{-1}$ na adição da amostra e na lavagem do cartucho e de $1 \mathrm{~mL}$ $\min ^{-1}$ na eluição, controlando-se por vácuo via manifold. Os extratos foram secos sob suave fluxo de gás nitrogênio e reconstituídos em $\mathrm{MeOH}$ (P-1 e P-2) e em ACN (P-3 e P-4). Utilizou-se água desionizada fortificada com solução-padrão mista dos estrógenos, em uma concentração inicial de $2,5 \mu \mathrm{g} \mathrm{L}^{-1}$ de cada hormônio. A eficiência dos procedimentos de extração foi medida através do percentual de recuperação, tendo sido aplicado o teste "t" de Student para comparação das médias.

\section{Volume de ruptura}

Após definição do adsorvente e do procedimento de extração, avaliou-se a capacidade máxima de adsorção, ou seja, o volume máximo de amostra que pode ser percolado para a concentração e separação dos analitos de interesse. Foram preparadas soluções aquosas (amostra testemunha) fortificadas com os analitos, mantendo-se
Tabela 2. Volume, concentrações e massa das soluções empregadas para determinação do volume de ruptura

\begin{tabular}{ccc}
\hline Volume $(\mathrm{mL})$ & Concentração $\left(\mu \mathrm{g} \mathrm{L}^{-1}\right)$ & Massa $(\mu \mathrm{g})$ \\
\hline 200 & 15,00 & \\
400 & 7,50 & \\
600 & 5,00 & 3,0 \\
800 & 3,75 & \\
1000 & 3,00 & \\
\hline
\end{tabular}

sempre a mesma massa das substâncias $(3,0 \mu \mathrm{g})$, alterando-se o volume de água potável e, conseqüientemente, a concentração (Tabela 2).

\section{Descloração}

Esta é uma etapa de substancial importância para prevenir a diminuição da concentração dos estrógenos foco deste estudo. Legalmente, água potável deve conter cloro residual, agente que previne contra contaminação microbiológica ${ }^{21}$ e também capaz de degradar hormônios estrógenos. ${ }^{22,23}$

Para verificação da necessidade da descloração, amostras-testemunhas contendo cloro residual $\left(1,5 \mathrm{mg} \mathrm{L}^{-1}\right)$ e outras descloradas com metabissulfito de sódio $\left(\mathrm{Na}_{2} \mathrm{~S}_{2} \mathrm{O}_{5}\right)$ foram fortificadas com $2,5 \mu \mathrm{g}$ $\mathrm{L}^{-1}$ de cada estrógeno e analisadas por CLAE-DAD.

Neste estudo, usou-se metabissulfito de sódio como agente neutralizador de cloro livre. Conforme descrito em Standard Methods, ${ }^{24}$ adicionou-se aos frascos de polietileno de alta densidade (PEAD), ainda antes da coleta, o equivalente a $0,1 \mathrm{~mL}$ de solução a $3 \%$ deste reagente, para cada $100 \mathrm{~mL}$ de amostra coletada.

\section{Análise cromatográfica}

As condições cromatográficas para separação dos estrógenos foram verificadas por meio da eluição dos analitos frente a diferentes fases móveis $\left(\mathrm{H}_{2} \mathrm{O}, \mathrm{ACN}, \mathrm{MeOH}\right)$, variando-se seus constituintes e proporção, $\mathrm{pH}$ e fluxo. Como ponto de partida, procedeu-se às análises conforme descrito em diferentes bibliografias pesquisadas, as quais foram modificadas ou desconsideradas no estudo, mediante os resultados encontrados. ${ }^{3,6,16,20,25}$ Foram verificadas fases móveis binárias empregando-se $\mathrm{ACN}, \mathrm{MeOH}$ e água desionizada em diferentes proporções; fluxos de 0,8 a 1,0 mL min $^{-1}$; e variação de $\mathrm{pH}$ da fase móvel aquosa.

O sistema cromatográfico utilizou CLAE (Varian Inc., ProStar 210) acoplado a DAD (Varian Inc., ProStar 335). Os esteróides foram separados empregando-se coluna Chromsep Microsorb 100 C18 (250 x 4,6 mm, $5 \mu \mathrm{m}$, Varian). A introdução da amostra no sistema foi manual com loop de $20 \mu \mathrm{L}$. 


\section{Validação}

O procedimento e os critérios para validação do método analítico proposto neste estudo seguiu as orientações do "Guia para Validação de Métodos Analíticos e Bioanalíticos" publicado pela Agência Nacional de Vigilância Sanitária (ANVISA), mediante a Resolução - RE $\mathrm{n}^{\circ} 899$, de 29 de maio de $2003 .{ }^{26}$

Os limites de detecção (LD) e inferior de quantificação (LIQ) foram determinados analisando-se a matriz contendo concentrações decrescentes do analito injetadas em quintuplicata. Os resultados obtidos foram comparados aos fornecidos pela análise da amostra branco. O LIQ correspondeu ao menor nível quantificável com precisão (20\%) e exatidão ( $80 \%$ a $120 \%)$ aceitáveis. Aquele que se apresentou como o menor nível detectável em comparação com o branco da matriz e que não satisfez as condições para ser considerado LIQ, foi identificado como LD do método.

Concomitantemente, a seletividade do método foi avaliada por meio da análise de 5 amostras da matriz fortificadas em concentração próxima ao LIQ e injetadas em triplicata, tendo sido os resultados comparados com aqueles obtidos das amostras em branco.

A curva analítica foi construída para cada estrógeno, utilizandose a mesma matriz proposta para o estudo, incluindo-se a análise de amostra em branco e de 6 amostras fortificadas com o padrão do analito, contemplando a faixa desde o LIQ até $120 \%$ da concentração mais alta a analisar. $\mathrm{O}$ cálculo da regressão linear pelo método dos mínimos quadrados foi usado para análise dos resultados, obtendo-se a equação da reta e o coeficiente de linearidade.

Para a validação da quantificação da substância de interesse e correção de um possível efeito da matriz, recorreu-se à adição de padrão; com isso se garantiu ainda a compensação de mudanças da resposta do equipamento, fornecendo melhor exatidão e precisão ao método como um todo. ${ }^{27}$

A precisão e a exatidão foram verificadas utilizando-se 3 concentrações (baixa, média e alta), contemplando a faixa linear dinâmica do método, com 5 repetições para cada nível. Estatisticamente, verificou-se a precisão, calculando-se o desvio padrão relativo (DPR). No ensaio de recuperação, os resultados analíticos de amostras fortificadas extraídas, com os obtidos a partir de soluções-padrão não extraídas. Os resultados encontrados foram submetidos ao teste " $\mathrm{t}$ " de Student em nível de 95\% de significância e 4 graus de liberdade, para comparação de médias.

\section{Análises de amostras coletadas no sistema de distribuição de água}

As amostras de água potável foram coletadas em abril de 2009, sendo duas na saída de duas ETAs distintas, uma em um reservatório de distribuição de água e outras duas na rede de distribuição de água, já em ponto disponível para uso do consumidor.

Em conjunto com as amostras em análise, foram preparadas quatro amostras de controle do método, denominadas "controle de qualidade" (CQ), de maneira a assegurar a correta execução deste e facilitar a identificação de algum erro durante alguma etapa da análise em curso. Uma das amostras foi fortificada no nível do LIQ (CQ-LIQ), uma em concentração baixa menor ou igual a três vezes menor o LIQ (CQB), outra em concentração igual à média aritmética entre CQB e a concentração alta (CQA) e a última em concentração alta equivalente à faixa de 75 a 90\% (CQA) do último nível da curva analítica.

\section{RESULTADOS E DISCUSSÃO}

As maiores dificuldades encontradas na separação cromatográfica foram localizar o pico do E3 em região de baixa interferência e estabelecer análise em tempo relativamente curto, apresentando picos com boa resolução (Rs).

$\mathrm{MeOH}$ foi testado como alternativa ao uso de acetonitrila (ACN) na fase móvel, porém após 25 min de análise nenhuma das substâncias foi eluída. Valores de $\mathrm{pH}(\approx 4,00 \mathrm{e} \approx 5,00)$ foram também testados, adicionando-se ácido acético glacial PA à fase móvel, resultando em supressão dos sinais analíticos. Igual modo foi a variação do fluxo de eluição que apenas alterou o tempo de retenção $\left(t_{R}\right)$ das substâncias sem, no entanto, oferecer melhores respostas.

A fase móvel selecionada consistiu de $\mathrm{ACN}$ e água, em modo gradiente, iniciando-se à proporção de 10:90 (ACN: $\left.\mathrm{H}_{2} \mathrm{O}\right)$, alterando-se para 50:50 em 5 min e, em seguida, para 54:46 ao longo dos $10 \mathrm{~min}$ seguintes, sendo esta última proporção mantida até o fim da corrida com duração total de $16,5 \mathrm{~min}$. O fluxo foi mantido em $1 \mathrm{~mL} \mathrm{~min}{ }^{-1}$ e o comprimento de onda em $280 \mathrm{~nm}$. Nestas condições, os picos apresentaram-se com boa resolução e sem interferências consideráveis.

Na Figura 1, é apresentada a sobreposição do cromatograma de uma amostra não-fortificada (branco) por um obtido a partir da injeção de solução-padrão mista na concentração de $3 \mathrm{mg} \mathrm{L}^{-1}$.

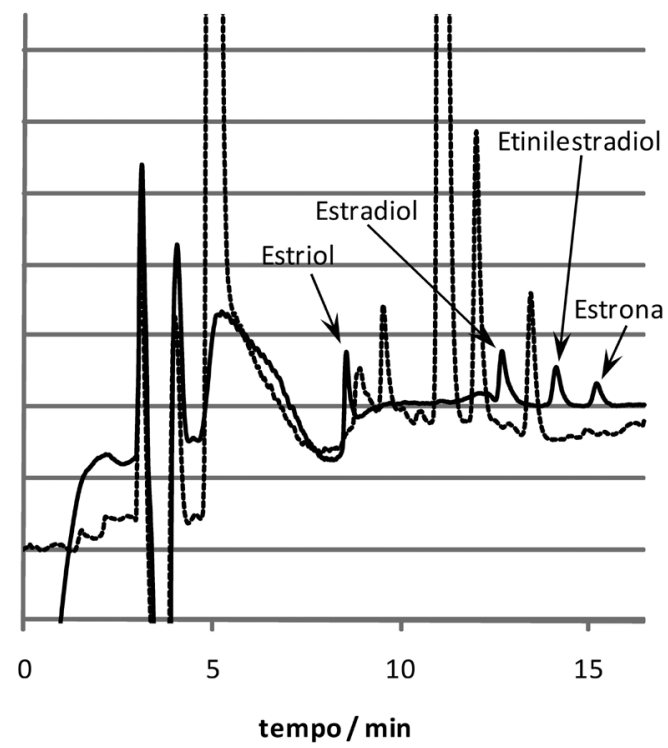

Figura 1. Sobreposição de cromatogramas, mostrando a não ocorrência de interferentes no $t_{R}$ dos analitos. Amostra branco não-fortificada (...) $e$ solução-padrão mista a $3 \mathrm{mg} \mathrm{L}{ }^{-1}$ (-). Condição usada: ACN: $\mathrm{H}_{2} \mathrm{O}$, em gra-

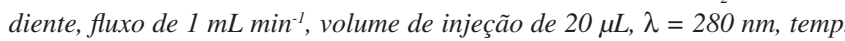
ambiente $27 \pm 0,5^{\circ} \mathrm{C}$

Na Figura 2 são comparadas as recuperações obtidas mediante uso de dois materiais adsorventes para cada procedimento de extração. Observase nitidamente que o adsorvente $\mathrm{C}_{18}$ apresentou menor dispersão entre os valores de recuperação, em relação aos poliméricos, principalmente os procedimentos P-1, com recuperações variando de $88,41 \%$ (estradiol) a 96,47\% (estriol); e P-2, de 76,81\% (estrona) a 95,02\% (estriol).

Para a ANVISA, é desejável que os valores de recuperação, na validação dos métodos voltados para análises de traços, sejam próximos a 100\%, admitindo-se valores menores, desde que não apresentem precisão e exatidão superiores a $20 \% .{ }^{26}$ Ribani e colaboradores ${ }^{27}$ afirmam que intervalos de recuperação entre 70 e $120 \%$ são geralmente aceitáveis, informação corroborada por Brito e colaboradores ${ }^{28}$ e Amarante Junior, Brito e Ribeiro. ${ }^{29}$ Portanto, os procedimentos $\mathrm{P}-1$, P-2 e P-4, empregando o adsorvente $\mathrm{C}_{18}$, e procedimento P-1 baseado em cartuchos com adsorvente polimérico (OPT), podem ser considerados adequados, enquanto os demais, por apresentarem resultados fora da faixa aceitável, são estatisticamente inadequados. 


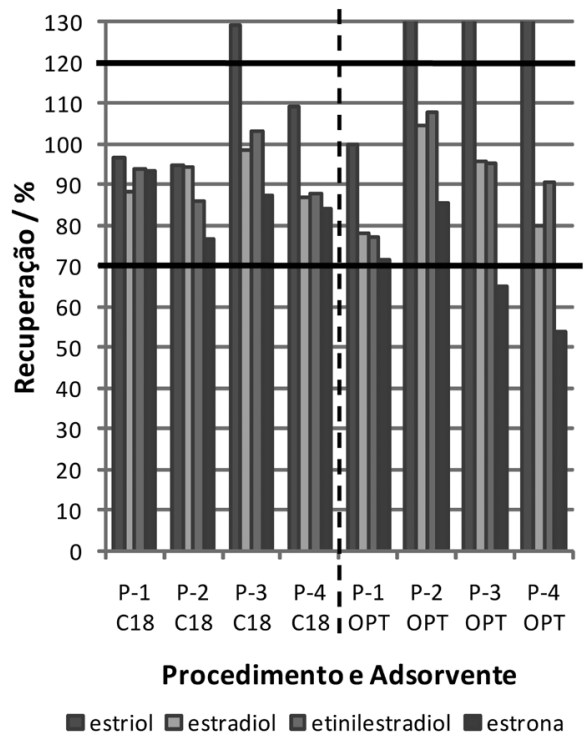

Figura 2. Comparação dos valores de recuperação entre adsorventes $C_{18}$ e OPT (polimérico), para os quatro procedimentos de extração (P-1, P-2, $P$-3 e P-4). As linhas em destaque correspondem ao valor máximo (120\%) e mínimo (70\%) aceitos para análises de resíduos

Dentre os procedimentos considerados adequados, $\mathrm{P}-1$ apresentou melhor recuperação; entretanto, a sua utilização pode tornar-se de custo elevado, face à atual escassez mundial do solvente acetonitrila. No ano passado, a pequena disponibilidade deste produto para comercialização fez seu preço aumentar até dez vezes, ${ }^{30}$ restando então como alternativa, o procedimento P-2 (uso do adsorvente $\mathrm{C}_{18}$ ), já que este apresentou desempenho similar ao P-4 (Tabela 1). Tal similaridade confirma-se pela comparação estatística das recuperações médias obtidas para cada procedimento. Foi empregado o teste de significância " $t$ " de Student, que resultou em valor de $t_{\text {calc }}=-1,125$, dentro da faixa de aceitação da igualdade entre as médias avaliadas $\left(-t_{\text {tab }}<t_{\text {calc }}<t_{\text {tab }}\right)$, sendo $t_{\text {tab }}=2,353$ e considerando 95\% $(\alpha=0,05$ e $v=0,025)$ de significância e $6\left(n_{1}+n_{2}-2\right)$ graus de liberdade.

Contrariamente às amostras com cloro residual, as descloradas apresentaram resultados semelhantes às soluções-padrão, fornecendo picos nos mesmos $t_{R}$ 's dos hormônios estrógenos (dados não mostrados). Na amostra que não foi tratada com metabissulfito de sódio foram identificados vários interferentes, além da ausência do sinal de alguns dos analitos investigados. Tais resultados indicaram a importância de se manter a descloração no preparo da amostra.

O fenômeno de desativação dos estrógenos por ação do cloro residual presente na água potável, que é oriundo da etapa de desinfecção ao término do tratamento da água, já era esperado, por se tratar de um processo oxidativo, um dos princípios que vêm sendo estudado por diversos pesquisadores para a eliminação da atividade estrogênica de diferentes substâncias. ${ }^{7,23,31}$ Conforme descreve Lopes, ${ }^{20}$ mesmo na análise imediata de água potável fortificada, foi observada uma redução na recuperação para um limite abaixo do mínimo aceitável. De acordo com Huber (2005) apud Lopes, ${ }^{20}$ os agentes desinfetantes mais comuns, como o dióxido de cloro e o cloro gasoso, reagem primeiramente com grupos funcionais que apresentam muitos elétrons, como fenóis e aminas, sendo, portanto, efetivos na oxidação de estrogênios quando aplicados no tratamento de água. Assim, esperase que tais reações de oxidação resultem na desarticulação do anel aromático presente nas moléculas dos estrógenos e, por conseguinte, na inativação de efeito estrogênico.

Hu e colaboradores ${ }^{32}$ submeteram solução aquosa de $17 \beta$-estradiol à cloração com hipoclorito de sódio sob condições controladas para avaliar a atividade estrogênica potencial devida àquela substância em água potável. Alguns produtos e subprodutos da oxidação foram identificados como sendo 2,4-dicloro-17 $\beta$-estradiol, 2,4-dicloroestrona, monocloroestrona e 4-[2-(2,6-dicloro-3-hidroxifenil) etil]$7 \alpha$-metiloctahidroinden-5-one. Observaram que a solução aquosa exposta a hipoclorito de sódio ainda apresentou, nos tempos de 120 e 180 min, atividade estrogênica cerca de $40 \%$ menor em comparação a esta mesma solução antes da cloração. Sugerem que tal ocorrência deve-se principalmente às substâncias 2,4-dicloro-17ß-estradiol e 2,4-dicloroestrona. Deborde et $\mathrm{al}^{33}$ estudaram, na presença de excesso de cloro total e sob condições controladas, a cinética de cloração de 6 desreguladores endócrinos em meio aquoso, dentre eles os 4 hormônios objetos deste estudo. Observaram que todas as moléculas constituídas com grupo fenólico em sua estrutura foram rapidamente oxidadas por ação do cloro. Relataram ainda que o tempo de meiavida, em pH 7, para os estrógenos avaliados variou de 6 a 8 min.

A Figura 3 ilustra o comportamento da recuperação obtida para cada volume de amostra e tipo de analito tratados. A linha horizontal em destaque indica o menor valor $(70 \%)$ considerado aceitável para a recuperação. Na avaliação dos resultados obtidos, considerou-se a dependência das recuperações para cada analito como condição limitante para a seleção do volume de amostra. Para 800 e $1000 \mathrm{~mL}$, os valores de recuperação foram inferiores à faixa adequada. No volume de $600 \mathrm{~mL}$, apenas estriol e estrona apresentaram resultados na faixa aceitável (70 a 120\%). Ao contrário, os volumes de 200 e 400 mL apresentaram recuperação adequada para todos os analitos. Tendo em vista conseguir-se maior fator de concentração e, consequentemente, menores LD e LIQ para o método, selecionou-se o volume de 400 $\mathrm{mL}$ como sendo o adequado para a pré-concentração da amostra.
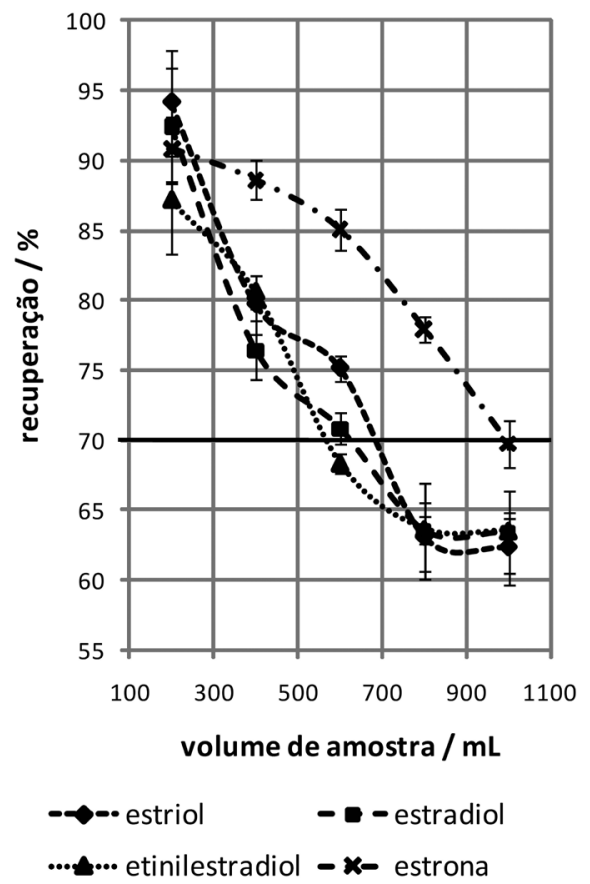

Figura 3. Avaliação do volume de ruptura, mediante aplicação do procedimento P-2, com cartucho C18 e 3 ug de cada substância

Sabendo que o extrato, após secagem em nitrogênio, foi reconstituído em 0,5 mL de $\mathrm{MeOH}$, o fator de concentração foi de 800 vezes. O tempo total de preparo da amostra para a análise cromatográfica foi aproximadamente de $4 \mathrm{~h}$, considerado aceitável para análise de rotina.

Os valores encontrados para $t_{\text {calc }}$ indicam que não há diferença estatisticamente significativa entre as recuperações obtidas e o valor 
desejado de $100 \%$, já que todos eles são menores do que o $t_{\text {tab }}(2,132)$. Ademais, observando-se os valores para exatidão e DPR, é possível afirmar que o método desenvolvido é exato e preciso. A ANVISA admite que o DPR seja $\leq 20 \%$ para o LIQ, não sendo superior a $15 \%$ para os demais níveis. ${ }^{26} \mathrm{O}$ método foi linear na faixa de 1,25 a 7,50 $\mu \mathrm{g}$ $\mathrm{L}^{-1}\left(\mathrm{LD}=0,0625 \mu \mathrm{g} \mathrm{L}^{-1}\right)$, para E3 e de 3,75 a 7,50 $\mu \mathrm{g} \mathrm{L}^{-1}(\mathrm{LD}=1,25$

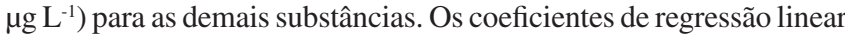
$\left(r^{2}\right)$ resultaram em valores maiores que 0,98 , e os DPRs de cada ponto das curvas de calibração também foram adequados (Figura 4). Tais resultados indicam que nesta faixa o método de separação cromatográfica é seguramente adequado para a determinação dos analitos de interesse. Na Tabela 3 são mostrados os resultados de recuperação, precisão e exatidão obtidos para cada substância estudada.

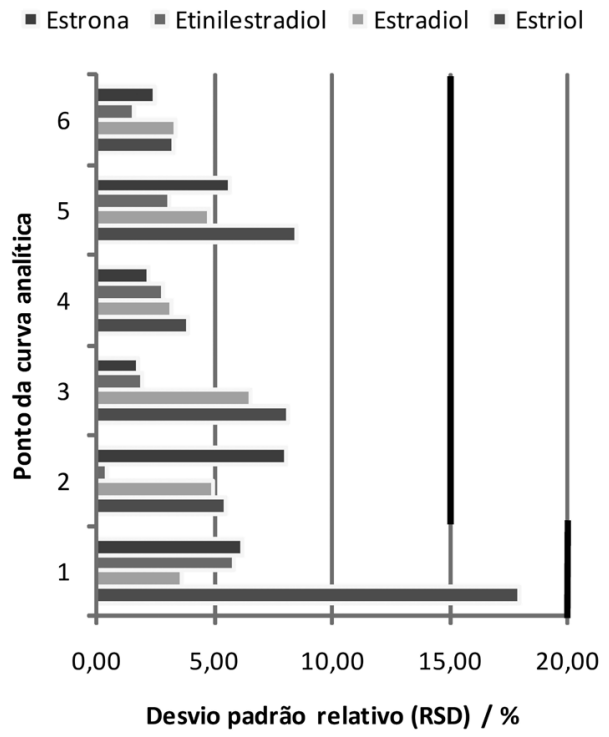

Figura 4. Flutuação dos DPRs calculados a partir das áreas obtidas na construção das curvas analíticas. As linhas em destaque representam o limite máximo para valores adequados

Observando-se a Figura 5, é possível notar que não foram detectados picos de nenhum dos hormônios estudados nas amostras analisadas. Entretanto, mesmo a Amostra 3 tendo apresentado picos próximos dos $t_{\mathrm{R}}$ 's do estradiol $(\approx 12,6 \mathrm{~min})$ e do etinilestradiol $(\approx 14,0$ min), estes não foram relativos a hormônios mediante a comparação dos espectros de absorção fornecidos por estes sinais. O fato de tal amostra resultar em mais picos interferentes que as demais pode estar relacionado com o aspecto visivelmente mais turvo por ela apresentado, ocorrência que não era esperada por se tratar de água potável. Já que este acontecimento foi isolado, pode-se atribuir à tubulação próxima ou à torneira do ponto de coleta que se apresentavam sujos. Para ter avaliação mais criteriosa quanto a isto, sugere-se que, em outro estudo, sejam avaliadas as influências das caracteríticas físicoquímicas da água sobre o método desenvolvido.

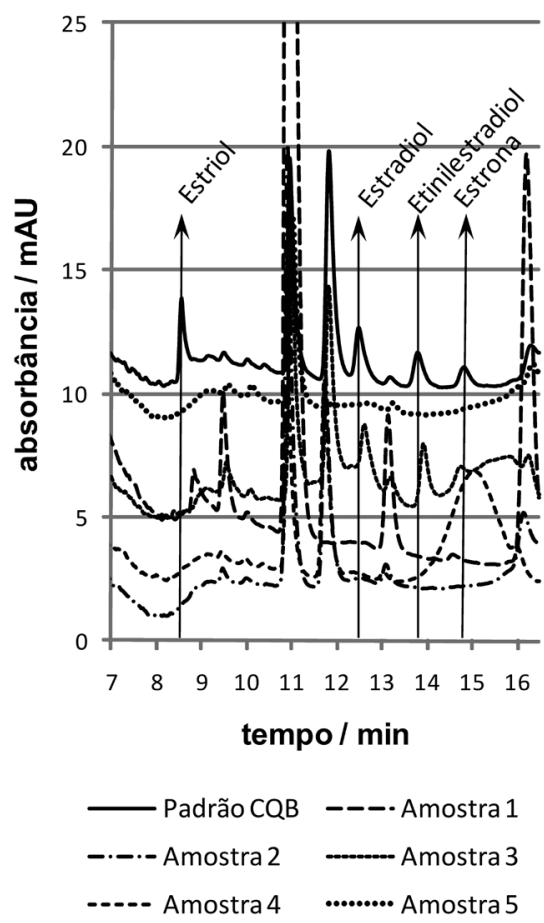

Figura 5. Sobreposição dos cromatogramas das 5 amostras analisadas e da amostra-testemunha fortificada em nível baixo $(C Q B)$

Finalmente, os limites de detecção e inferior de quantificação do método não foram suficientemente baixos para a eficiente determinação destas substâncias em água potável, pois conforme estudos já publicados, ${ }^{9,16,20,34}$ estas substâncias têm potencial presença em

Tabela 3. Resultados de recuperação, precisão e exatidão obtidos para cada hormônio estrógeno em três níveis de fortificação e cinco repetições (n=5)

\begin{tabular}{|c|c|c|c|c|c|c|}
\hline Substância & Nível & Concentração $\left(\mu \mathrm{g} \mathrm{L}^{-1}\right)$ & Recuperação a (\%) & $\mathrm{t}_{\text {calc }}{ }^{\mathrm{b}}$ & $\operatorname{DPR}^{\mathrm{c}}(\%)$ & Exatidão (\%) \\
\hline \multirow[t]{3}{*}{ Estriol } & baixo & 1,25 & $94(80-119)$ & $-0,6870$ & 19,40 & 109,41 \\
\hline & médio & 3,75 & $85(77-91)$ & $-5,3908$ & 0,07 & 99,22 \\
\hline & alto & 6,25 & $98(87-104)$ & $-0,8137$ & 0,06 & 99,66 \\
\hline \multirow[t]{3}{*}{ Estradiol } & baixo & 3,75 & $73(70-75)$ & $-34,3382$ & 2,41 & 100,62 \\
\hline & médio & 5,00 & $84(77-89)$ & $-7,0292$ & 6,34 & 96,73 \\
\hline & alto & 6,25 & $103(95-109)$ & 1,2753 & 5,44 & 101,69 \\
\hline \multirow[t]{3}{*}{ Etinilestradiol } & baixo & 3,75 & $81(70-91)$ & $-4,8067$ & 11,22 & 94,96 \\
\hline & médio & 5,00 & 107 (101-117) & 1,8603 & 6,27 & 102,95 \\
\hline & alto & 6,25 & $104(100-105)$ & 2,0367 & 3,11 & 100,09 \\
\hline \multirow[t]{3}{*}{ Estrona } & baixo & 3,75 & $96(87-105)$ & $-1,4606$ & 6,80 & 96,26 \\
\hline & médio & 5,00 & $101(91-107)$ & 1,4907 & 6,56 & 91,52 \\
\hline & alto & 6,25 & $104(97-107)$ & 1,3204 & 5,79 & 104,69 \\
\hline
\end{tabular}

${ }^{\mathrm{a}}$ Corresponde à média aritmética dos valores e entre parênteses os valores mínimo e máximo, respectivamente, ${ }^{\mathrm{b}}$ “ $\mathrm{t}$ ”de Student, ${ }^{\mathrm{c}}$ desvio padrão relativo (precisão) 
água potável, o que exige o seu monitoramento mediante métodos analíticos que cubram níveis traço.

\section{CONCLUSÃO}

O estudo possibilitou a separação cromatográfica dos hormônios estriol (E3), $17 \beta$-estradiol (E2), estrona (E1) e $17 \alpha$-etinilestradiol (EE2) em tempo relativamente curto. O preparo da amostra, incluindo a descloração e a extração em fase sólida (EFS), resultou em valores de recuperação dentro do limite aceitável.

O método desenvolvido foi considerado validado, após avaliação dos parâmetros: seletividade; linearidade; precisão; exatidão; limite inferior de quantificação; limite de detecção; recuperação e repetitividade, mostrando-se, portanto, seletivo, linear, exato e preciso para o procedimento de preparo da amostra e as condições cromatográficas estabelecidas.

Durante a aplicação do método validado às análises de amostras de água potável coletadas, observou-se que o mesmo não foi capaz de identificar ou quantificar hormônios estrógenos, no nível de sensibilidade exigido, mas existe a possibilidade de realmente os hormônios não estarem presentes na água potável, na época da coleta. Sabendo-se que o cloro residual presente pode degradar as substâncias estrógenas ${ }^{11,32,33,35}$ e que, antes de chegar à rede de distribuição, a água potável ao sair de uma das ETAs percorre mais de $56 \mathrm{Km}$ de tubulação (tempo de detenção $\approx 8 \mathrm{~h}$ ), pode-se também supor a sua degradação durante o deslocamento em função do contato com cloro residual. Desta forma, é necessário pesquisar as águas naturais superficiais que são captadas para o tratamento, podendo-se assim investigar a remoção destas substâncias pelo processo de tratamento das ETAs.

\section{REFERÊNCIAS}

1. Chang, H.-S.; Choo, K.-H.; Lee, B.; Choi, S.-J.; J. Hazard. Mater. 2009, $172,1$.

2. Reis Filho, R. W.; Araújo, J. C.; Vieira, E. M.; Quim. Nova 2006, 29, 817.

3. Araújo, J. C.; Dissertação de Mestrado, Universidade de São Paulo, Brasil, 2006.

4. Johnson, A. C.; Williams, R. J.; Environ. Sci. Technol. 2004, 38, 3649; Gomes, R. L.; Scrimshaw, M. D.; Nester, J. N.; Trends Anal. Chem. 2003, 22, 697; Ghiselli, G.; Jardim, W. F.; Quim. Nova 2007, 30, 695.

5. Hansen, P.-D.; Dizer H.; Hock, B.; Marx, A.; Sherry, J.; McMaster, M.; Blaise, Ch.; Trends Anal. Chem. 1998, 17, 448.

6. Alda, M. J. L.; Barceló, D.; J. Chromatogr., A 2001, 911, 203.

7. Bila, D. M.; Dezotti, M.; Quim. Nova 2007, 30, 651.

8. Boyd, G. R.; Reemtsma, H.; Grimm, D. A.; Mitra, S.; Sci. Total Environ. 2003, 311, 135.

9. Rodriguez-Mozaz, S.; Alda, M. J. L.; Barceló, D.; J. Chromatogr., A 2004, 1045, 85.

10. Brasil, Ministério das Cidades; Sistema Nacional de Informações sobre Saneamento: visão geral da prestação dos serviços de água e esgotos 2004. MCIDADES. SNSA: Brasília, 2005; Reis Filho, R. W.; Barreiro, J. C.; Vieira, E. M.; Cass, Q. B.; Revista Ambi-Água 2007, 2, 54.

11. Chen, C.-Y.; Wen, T.-Y.; Wang, G.-S.; Cheng, H.-W.; Lin, Y.-H.; Lien, G.-W.; Sci. Total Environ. 2007, 378, 352.

12. Liu, Z.-H.; Kanjo, Y.; Mizutani, S.; Sci. Total Environ. 2009, 407, 731.

13. Aerni, H.-R.; Kobler, B.; Rutishauser, B. V.; Wettstein, F. E.; Fischer, R.; Giger, W.; Hungerbühler, A.; Marazuela, M. D.; Peter, A.; Schönen- berger, R.; Vögeli, A. C.; Suter, M. J.-F. ; Eggen, R. L.; Anal. Bioanal. Chem. 2004, 378, 688; Lee, Y.; Gunten, U. V.; Environ. Sci. Technol. 2009, 43, 480; Kuster, M.; Azevedo, D. A.; Alda, M. J. L.; Aquino Neto, F. R.; Barceló, D.; Environ. Int. 2009, 35, 997.

14. Sanfilippo, K.; Pintoa, B.; Colombinib, M. P.; Bartoluccib, U.; Realia, D.; J. Chromatogr., B 2010, 878, 1190; Balsiger, H. A.; Torre, R.; Lee, W.-Y.; Cox. M. B.; Sci. Total Environ. 2010, 408, 1422.

15. Palumbo, A. J.; Koivunenb, M.; Tjeerdem, R. S.; Sci. Total Environ. 2009, 407, 953; Gadd, J. B.; Tremblay, L. A.; Northcott, G. L.; Environ. Pollut. 2010, 158, 730.

16. Ghiselli, G.; Tese de Doutorado, Universidade Estadual de Campinas, Brasil, 2006

17. Sodré, F. F.; Montagner, C. C.; Locatelli, M. A. F.; Jardim, W. F.; J. Braz. Soc. Ecotoxicol. 2007, 2, 187.

18. Silva, R. N. M. da; Gomes, L. V.; Freitas, M. I. C.; Gedeon, A. D.; Duarte, M. do C. R.; Resumos do $23^{\circ}$ Congresso Brasileiro de Engenharia Sanitária e Ambiental, Campo Grande, Brasil, 2005.

19. Pinheiro Júnior, J. de R.; Costa, L. A. da; Santos, M. C. F. V. dos; Gomes, L. N.; Anais do $13^{\circ}$ Simpósio Brasileiro de Sensoriamento Remoto, Florianópolis, Brasil, 2007.

20. Lopes, L. G.; Tese de Doutorado, Universidade Estadual Paulista, Brasil, 2007.

21. Heller, L.; Pádua, V. L.; Abastecimento de água para consumo humano, UFMG: Belo Horizonte, 2006.

22. Bila, D. M.; Montalvao, A. F.; Azevedo D. de A.; Dezotti, M.; Chemosphere 2007, 69, 736.

23. Ternes, T. A.; Stüber, J.; Herrmann, N.; McDowell, D.; Ried, A.; Kampmann, M.; Teiser, B.; Water Res. 2003, 37, 1976; Roloff, G. A.; Dissertação de Mestrado, Universidade de São Paulo, Brasil, 2007.

24. Clescerl, L. S. Em Standard Methods for the Examination of Water and Wastewater, $20^{\text {th }}$ ed.; Greenberg, A. E.; Eaton, A. D., eds.; WEF, APHA, AWWA: Maryland, 1998.

25. Alda, M. J. L.; Barceló, D.; J. Chromatogr., A 2000, 892, 391; Alda, M. J. L.; Diaz-Cruz, S.; Petrovic, M.; Barceló, D.; J. Chromatogr., A 2003, 1000, 503; Rodriguez-Mozaz, S.; Alda, M. J. L.; Barceló, D.; J. Chromatogr., A 2004, 1045, 85.

26. Agência Nacional de Vigilância Sanitária (ANVISA); Resolução RE $n^{o}$ 899, de 29/05/2003.

27. Ribani, M.; Bottoli, C. B. G.; Collins, C. H.; Jardim, I. C. S. F.; Melo, L. F. C.; Quim. Nova 2004, 27, 771.

28. Brito, N. M.; Amarante Júnior, O. P. de; Polese, L.; Ribeiro, M. L.; Pesticidas: Revista de Ecotoxicologia e Meio Ambiente 2003, 13, 129.

29. Amarante Júnior, O. P. de; Brito, N. M.; Ribelo, M. L.; Pesticidas: Revista de Ecotoxicologia e Meio Ambiente 2005, 15, 15.

30. Lanças, F.M.; Scientia Chromatographica 2009, 1, 51.

31. Bila, D. M.; Tese de Doutorado, Universidade Federal do Rio de Janeiro, Brasil, 2005; Schäfer, A. I.; Nghiem, L. D.; Waite, T. D.; Environ. Sci. Technol. 2003, 37, 182.

32. Hu, J.; Cheng, S.; Aizawa, T.; Terao, Y.; Kunikane, S.; Environ. Sci. Technol. 2003, 37, 5665.

33. Deborde, M.; Rabouan, S.; Gallard, H.; Legube, B.; Environ. Sci. Technol. 2004, 38, 5577.

34. Kuch, H. M.; Ballschmiter, K.; Environ. Sci. Technol. 2001, 35, 3201; Aherne, G. W.; Briggs, R.; J. Pharm. Pharmacol. 1989, 41, 735.

35. Koh, Y. K. K.; Chiu, T. Y.; Boobis, A.; Cartmell, E.; Scrimshaw, M. D.; Lester, J. N.; Environ. Technol. 2008, 29, 245. 